\title{
The density profiles of dark matter halos in spiral galaxies
}

\author{
Gianluca Castignani $^{1,2}$, Noemi Frusciante $^{1,2}$, Daniele Vernieri $^{12^{*}}$, Paolo Salucci $^{1,2}$ \\ ${ }^{1}$ SISSA-ISAS, International School for Advanced Studies, Trieste, Italy; ${ }^{*}$ Corresponding Author: daniele.vernieri@sissa.it \\ ${ }^{2}$ INFN, Istituto Nazionale di Fisica Nucleare, Sezione di Trieste, Trieste, Italy
}

Received 19 January 2012; revised 18 February 2012; accepted 5 March 2012

\begin{abstract}
In spiral galaxies, we explain their non-Keplerian rotation curves (RCs) by means of a non-luminous component embedding their stellar-gaseous disks. Understanding the detailed properties of this component (labelled Dark Matter, DM) is one of the most pressing issues of Cosmology. We investigate the recent relationship (claimed by Walker et al. 2010, hereafter $\mathrm{W}+10$ ) between $r$, the galaxy radial coordinate, and $V_{h}(r)$, the dark halo contribution to the circular velocity at $r, 1$ ) in the framework of the Universal Rotation Curve (URC) paradigm and directly 2) by means of the kinematics of a large sample of DM dominated spirals. We find a general agreement between the $W+10$ claim, the distribution of DM emerging from the URC and that inferred in the (low luminosity) objects of our sample. We show that such a phenomenology, linking the spiral's luminosity, radii and circular velocities, implies an evident inconsistency with (naive) predictions in the $\Lambda$ Cold Dark Matter (^CDM) scenario.
\end{abstract}

Keywords: Galaxies; Spiral; Kinematics and Dynamics; Dark Matter

\section{INTRODUCTION}

Rotation curves (RCs) of spiral galaxies show no asymptotic Keplerian behavior and fail to match the distribution of the luminous matter. The favored explanation is the existence of an unknown unseen component, the Dark Matter (DM): the "luminous" components of galaxies such as gas and stars are distributed inside a spherical "dark" halo".

Recently, [2], hereafter $\mathrm{W}+10$, following the pioneer paper of [3] $(\mathrm{M}+07)$, found that the dark component of the circular velocity (and of mass) profile shows a characteristic and similar behavior both in Spirals (and in Milky Way dSphs) of very different luminosities. More

${ }^{1} \mathrm{~A}$ thorough review on this issue can be found in [1] and downloaded at www.sissa.it/ap/dmg/dmaw presentation.html. precisely, they investigated a sample of 60 spirals, whose RCs span radii from $1 \mathrm{kpc}$ to $75 \mathrm{kpc}$, have maximum amplitudes in the range $50 \mathrm{~km} \cdot \mathrm{s}^{-1} \leq V_{\max } \leq 300 \mathrm{~km} \cdot \mathrm{s}^{-1}$ and their baryonic masses $M_{b}$ ranging between $3 \times 10^{8}$ $\mathrm{M}_{\odot}$ and $4 \times 10^{11} \quad \mathrm{M}_{\odot}$. They derived $V_{h}(r)$, the halo contributions to the circular velocity (in short the "halo RCs"), by subtracting the contribution of the baryonic matter from the circular velocity: $V_{h}^{2}(r)=V^{2}(r)-V_{b}^{2}(r)$ (see $\mathrm{W}+10$ for details). They found a correlation between $V_{h}(r)$ and $r($ see $\mathrm{W}+10)$ :

$$
\log \left(\frac{V_{h}(r)}{\mathrm{km} \cdot \mathrm{s}^{-1}}\right)=1.47_{-0.19}^{+0.15}+0.5 \log \left(\frac{r}{\mathrm{kpc}}\right)
$$

independently of galaxy luminosity. Moreover:

$\frac{M_{h}(r)}{M_{\odot}}=2_{-1.2}^{+2} \times 10^{8}\left(\frac{r}{\mathrm{kpc}}\right)^{2}$. Noticeably, at $r=R_{1 / 2}$, being $R_{1 / 2}$ the radius encompassing half of the stellar mass, the relation (1) can be investigated also in dwarf spheroidals, and found to hold also in these systems (see $\mathrm{W}+10$ for details).

These are surprising findings: in fact, when, in objects of very different luminosity we derive their $V_{h}(r)$ (the dark halo-contribution to the circular velocities) and plot them as a function of radius, a well defined curve emerges, instead of a more likely scatter plot. On the other hand, the circular velocities $V(r)$ show a very different phenomenology. From the analysis of several thousands of RCs, an Universal Rotation Curve (URC) emerges, i.e. a specific 3-dimensional surface linking 1) the circular velocity at a normalized radius $r / R_{D}$ (i.e. measured in units of disk length-scale), 2) the galaxy luminosity and 3 ), the normalized radius (see [4,5] (PSS), [6-8] (S + 07) for details).

Summarizing, in the intermediate regions of spirals, W +10 found: $V_{h}(r)=F_{\mathrm{W}+10}(r)$, independently of galaxy luminosity $L$ and stellar disk length-scale $R_{D}$. In the URC scenario, instead, we have: $V_{h}(r)=F_{U R C}\left(r / R_{D}, L\right)$, i.e. the halo component of the circular velocity is a function of disk luminosity and disk length scale. Let us stress that the existence of the URC implies that the structural parameters of the Dark and Luminous components are strongly related, but not necessarily, through the very 
constraining Eq.1.

In this paper, we investigate whether these two apparently different results are compatible. We also use the outcome to derive important information on the DM distribution in spirals. We will study an independent (larger) sample of spirals by means of reliable method of mass modelling. From [9] (PS95) we select 116 spiral galaxies (hereafter Sample A) whose RCs satisfy the following requirements: a) no presence of a prominent bulge:

$V\left(0.2 R_{\text {opt }}\right)<50 \mathrm{~km} \cdot \mathrm{s}^{-12}$, or if the latter is missing: $\left.V\left(0.4 R_{\text {opt }}\right)<70 \mathrm{~km} \cdot \mathrm{s}^{-1}, \mathrm{~b}\right)$ low luminosity objects:

$V(r)<120 \mathrm{~km} \cdot \mathrm{s}^{-1}$, at any radius, that implies:

$M_{I}>-20.2$, with $M_{I}$ the magnitude in the I band, c) the RCs have a measure either at $0.8 R_{o p t}$ or at $1.0 R_{\text {opt }}$.

Let us notice that we limit ourselves to objects of low luminosity, in that they are DM dominated (e.g. [11] (PS90)) and therefore $V_{h}(r)$ can be derived with great accuracy. Higher luminosity spirals are disk dominated and therefore $V_{h}(r)$ strongly depends on the details and on the assumptions in the mass modelling. The RCs of Sample A have a radial range matching that of $\mathrm{W}+10$ sample $\max \left[\frac{1}{3} R_{D}, 1 \mathrm{kpc}\right] \leq r \leq 6 R_{D}$. Following $\mathrm{W}+10$, we adopted $1 \mathrm{kpc}$ as the innermost radius in that, inside which, the stellar disk (sometimes with a stellar bulge) dominates the gravitational potential making difficult the estimate of $V_{h}(r)$.

Let us compare the $\mathrm{W}+10$ relationship with the URC analogue (PSS, S + 07) and with that we derive for Sample A. From the condition of centrifugal equilibrium we have:

$$
V^{2}(r)=V_{\text {lum }}^{2}(r)+V_{h}^{2}(r)
$$

where the subscripts lum and $h$ stand respectively for luminous and halo components. The former is the quadratic sum of disk, bulge and gas contributions. Here, we neglect the latter two because 1) the selected low luminosity objects have, for $r>1 \mathrm{kpc}$, a negligible bulge, 2) the gaseous disk is important only for $r>R_{o p t}$, i.e. outside the region that we are considering here (see [12]). For the objects of Sample A, each RC has 2 - 4 independent measurements (see Table 2 of PS95) and therefore the same number of halo velocity data. For the URC, there is analytical formula for $V_{h}(r)$ (see below). Hereafter, we adopt a flat cosmology with matter density parameter $\Omega_{M}=0.27$ and Hubble constant $H_{0}=75 \mathrm{~km} \cdot \mathrm{s}^{-1} \cdot \mathrm{Mpc}^{-1}$.

\section{THE CONTRIBUTION OF DARK MATTER HALOS TO THE CIRCULAR VELOCITY OF SPIRALS}

We derive the velocity halo contribution for the objects

${ }^{2} R_{\text {opt }}$ is the radius encircling $83 \%$ of the light, for a Freeman exponential disk $R_{o p t}=3.2 R_{D}$ where $R_{D}$ is the disk scale-length, see [10]. of Sample A in the following way; we assume a Freeman profile to describe the stellar disk surface density, then [10]:

$$
V_{D}^{2}(x)=14.9 \beta(x) V^{2}(1.0) x^{2}\left[I_{0} K_{0}-I_{1} K_{1}\right]_{1.6 x}
$$

where $x=r / R_{o p t}, I_{n}$ and $K_{n}$ are the modified Bessel functions. Let us also notice that: $R_{1 / 2}=1.67 / 3.2 R_{o p t}$ $=0.52 R_{\text {opt }}$. The mass modelling of individual (and coadded) RCs of objects of low luminosity (as those in Sample A) is rather simple. We have: $\beta(1) \simeq 0.13$ and $\beta(0.8) \simeq 0.23$ (see PS90 and PSS). More precisely, there is a small luminosity dependence of $\beta$ but for the objects in Sample A this is irrelevant. Then, for any object, we derive $V_{h}(r)$ by subtracting the (small) disk contribution $V_{D}^{2}(r)$, given by Eq.3, from the circular velocity $V^{2}(r)$. Finally, we derive $V_{h}\left(R_{1 / 2}\right)$ by linearly interpolating $V_{h}\left(0.4 R_{o p t}\right)$ and $V_{h}\left(0.6 R_{o p t}\right)$.

The URC leads to an (analytical) Universal form for $V_{h}(r)$, i.e. for the halo velocity component (URCH) to $V_{U R C}(r)$ which was built by coadding the kinematics of thousands of galaxies (PSS, S + 07). We model the URC, that represents the typical $\mathrm{RC}$ of an object of magnitude $M_{I}$, (or of virial halo mass $M_{v i r}$ (see $\mathrm{S}+07$ and reference therein) in its dark and luminous components $\left.(\text { e.g. } \mathrm{S}+07)^{3}\right)$.

The $\mathrm{W}+10$ halo velocity-radius relationship (black line) with its $1-\sigma$ scatter (black dashed lines) compared with the halo velocity calculated at $R_{1 / 2}$ as function of $R_{1 / 2}$ for 1) our Sample A whose points are calculated by interpolating $V_{h}\left(0.4 R_{\text {opt }}\right)$ and $V_{h}\left(0.6 R_{\text {opt }}\right)$ for each galaxy (black points) and 2) URCH profiles (red line).

In detail the URC DM density profile is

$$
\rho_{U R C H}(r)=\frac{\rho_{0} r_{0}^{3}}{\left(r+r_{0}\right)\left(r^{2}+r_{0}^{2}\right)}
$$

where $r_{0}$ is the core radius and $\rho_{0}$ is the central halo density. It follows that:

$$
\begin{aligned}
V_{U R C H}^{2}(r)= & 6.4 \frac{\rho_{0} r_{0}^{3}}{r}\left[\ln \left(1+\frac{r}{r_{0}}\right)-\arctan \left(\frac{r}{r_{0}}\right)\right. \\
& \left.+\frac{1}{2} \ln \left(1+\frac{r^{2}}{r_{0}^{2}}\right)\right]
\end{aligned}
$$

From [14] we have:

$$
\rho_{0}=5 \times 10^{-24}\left(r_{0} /(8.6 \mathrm{kpc})\right)^{-1} \mathrm{~g} \cdot \mathrm{cm}^{-3}
$$

and

$$
\log \left(\frac{r_{0}}{\mathrm{kpc}}\right) \simeq 0.66+0.58 \log \left(\frac{M_{v i r}}{10^{11} M_{\odot}}\right)
$$

\footnotetext{
${ }^{3}$ The virial mass $M_{v i r}$ corresponds to the mass enclosed in a sphere with density $101 \rho_{c}$, being $\rho_{c}$ the critical density of the Universe. $M_{v i r}$ and the virial radius are related by $R_{v i r}=259\left(M_{v i r} / 10^{12} M_{\odot}\right)^{1 / 3} \mathrm{kpc}$, see [13].
} 
The disk length-scales and masses, $R_{D}$ and $M_{D}$, are related to the halo masses through (see [15] and $\mathrm{S}+07$ )

$$
\begin{aligned}
\log \left(\frac{R_{D}}{\mathrm{kpc}}\right)= & 0.633+0.379 \log \left(\frac{M_{D}}{10^{11} M_{\odot}}\right) \\
& +0.069\left(\log \frac{M_{D}}{10^{11} M_{\odot}}\right)^{2}
\end{aligned}
$$

and

$$
M_{D}=2.3 \times 10^{10} M_{\odot} \frac{\left[M_{v i r} / 3 \times 10^{11} M_{\odot}\right]^{3.1}}{1+\left[M_{v i r} /\left(3 \times 10^{11} M_{\odot}\right)\right]^{2.2}}
$$

By means of Eqs.4-9 we can derive, the URCH halo velocity $V_{h}^{U R C}\left(r / R_{D}, L\right)$.

The first step is to investigate the $V_{h}\left(R_{1 / 2}\right)-R_{1 / 2}$ relationship, that holds also for objects of different Hubble types. In Figure 1 we plot the URCH as a red line and the relation for Sample A as black points. It is clear that both individual and URC halo velocities do correlate with $R_{1 / 2}$, well matching the $\mathrm{W}+10(\mathrm{M}+07)$ relation (shown as black line).

The further step is to investigate the full profile $V_{h}(r)$ at "intermediate radii" $\max \left[\frac{1}{3} R_{D}, 1 k p c\right] \leq r \leq 6 R_{D}$. Each of the 116 spirals contributes with $2-4$ independent kinematical measurements. In Figure 2 we plot $V_{h}(r)$ obtained from the RCs of Sample A, once that the luminous component has been removed as explained above.

The $\mathrm{W}+10$ halo velocity-radius relationship (black line) with its $1-\sigma$ scatter (black dashed lines) plotted with 1) the halo velocities of our Sample A of 116 spirals (black points) (PSS, PS90), 2) the URCH profiles (red lines), corresponding to objects with mass comparable with those in the $\mathrm{W}+10$ sample. The error (of 20\%) in the individual determination of $V_{h}$ is shown as an errorbar.

We also plot, as two red lines, the URCH velocity profiles of spirals with luminosities similar to those of the objects of Sample $A^{4}$, and to the great majority of the 60 spirals of the $\mathrm{W}+10$ sample. Notice that, given the Schechter-like form of the luminosity function of spirals, in the $\mathrm{W}+10$ sample there are only an handful of big galaxies.

In the low luminosity range we plot $V_{h}(r)$ for the individual RCs of Sample A (black points) and for the corresponding URCH profiles, and compare them with the $\mathrm{W}+10$ relationship. Let us recall that we did not investigate the individual $V_{h}(r)$ of luminous spirals since their dark-luminous RC decomposition is quite uncertain. However, we have investigated the high luminosity objects by means of the URCH, that we show in Figure 3 alongside with the $\mathrm{W}+10$ relationship. Summarizing,

${ }^{4}$ The related virial masses are $M_{v i r} / M_{\odot}=1.17 \times 10^{11}, 7.7 \times 10^{11}$.

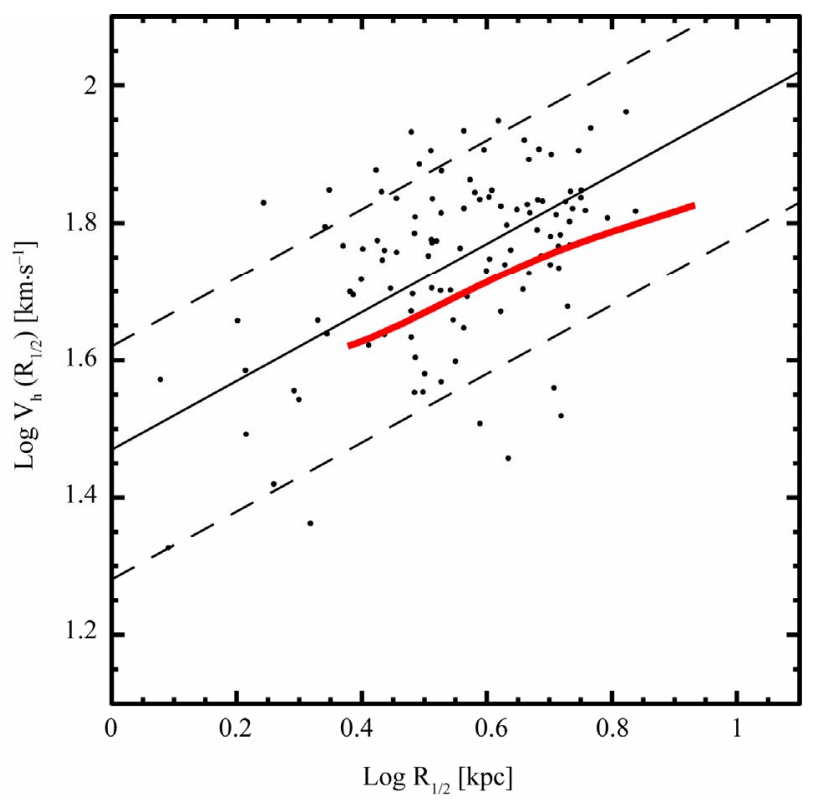

Figure 1. The $\mathrm{W}+10$ halo velocity-radius relationship (black line) with its 1-s scatter (black dashed lines) compared with the halo velocity calculated at $R_{1 / 2}$ as function of $R_{1 / 2}$ for 1 ) our Sample A whose points are calculated by interpolating $V_{h}\left(0.4 R_{\text {opt }}\right)$ and $V_{h}\left(0.6 R_{o p t}\right)$ for each galaxy (black points) and 2) URCH profiles (red line).

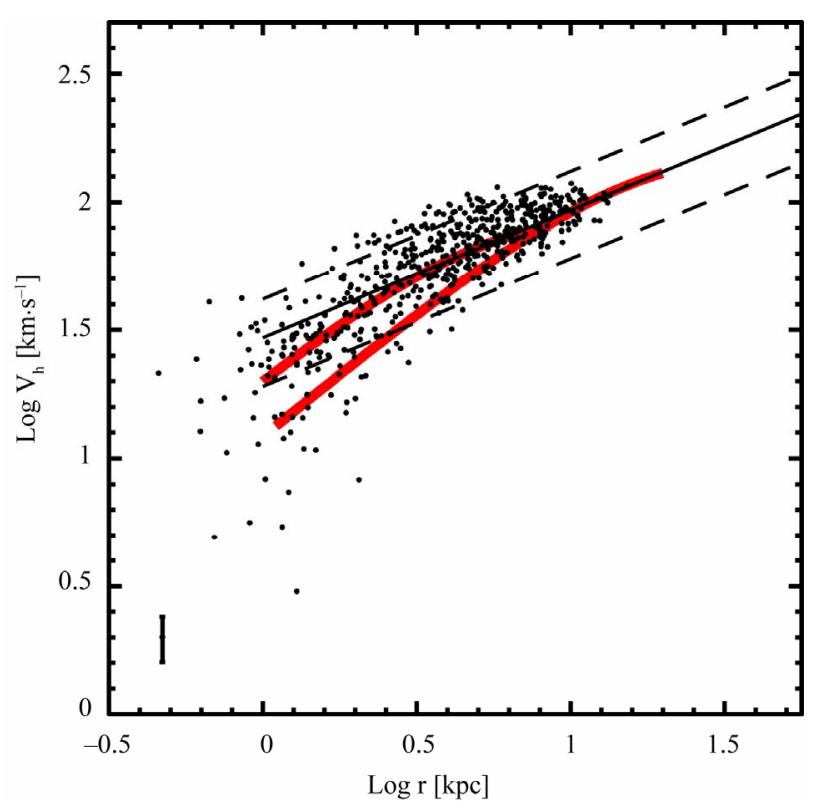

Figure 2. The $\mathrm{W}+10$ halo velocity-radius relationship (black line) with its 1-s scatter (black dashed lines) plotted with 1) the halo velocities of our Sample A of 116 spirals (black points) (PSS, PS90), 2) the URCH profiles (red lines), corresponding to objects with mass comparable with those in the $\mathrm{W}+10$ sample. The error (of 20\%) in the individual determination of $V_{h}$ is shown as an errorbar.

for spirals in halos with $M_{v i r}>1.9 \times 10^{12} M_{\odot}$, we derive $V_{h}(r)$ by means of the URCH, for halos of lower mass, 
instead, we derive $V_{h}(r)$ from 1) the URCH (red lines) and 2) the mass modelling of the individual RCs of Sample A (black points). In both cases, the (derived) halo velocities are compared with the $\mathrm{W}+10$ relationship.

\section{UNIVERSAL HALO VELOCITY PROFILE AND IMPLICATIONS}

We find for Sample A and also from the URC, that: $V_{h}\left(R_{1 / 2}\right) \propto R_{1 / 2}^{0.5}$, very similar to the $\mathrm{W}+10$ relation. This result is not a new one, it arises as effect of the particular dependencies of the dark and luminous structural parameters on luminosity and disk length scale. We can show this by looking at the Radial Tully Fisher relation ([16]). From their Eq.9 one has $L_{I} \propto V\left(R_{1 / 2}\right)^{2.55}$, where $L_{I}$ is the I-band luminosity, considering that for spirals we have: $R_{1 / 2} \propto R_{D} \propto L^{0.5}$, and that from the mass modelling of individual objects $V\left(R_{1 / 2}\right) / V_{h}\left(R_{1 / 2}\right) \propto V_{h}\left(R_{1 / 2}\right)^{0.2}$ (see PS90, [17,18], i.e. smaller galaxies have a larger fraction of DM), one arrives to $V_{h}\left(R_{1 / 2}\right) \propto R_{1 / 2}^{k}$, with $k$ very near to the value of +0.5 , claimed by $\mathrm{W}+10$ (see Figure 1).

However, with the complete data from the URC shown in Figure 4 we realize that $V_{h}(r)$ in Spirals of different luminosities, has not an unique profile, neither always it is a power law with exponent $1 / 2$. However, at low luminosities, the halo velocity individual contributions and the URCH profiles are in good agreement with the $\mathrm{W}+10$ result (see also $\mathrm{M}+07$ ).

At highest luminosities (see Figure 4) the $\mathrm{W}+10$ relationship cannot be reproduced by the URCH profiles. However let us stress that in these cases $V_{h}(r)$ depend significantly (especially in the $\mathrm{W}+10$ work) on the

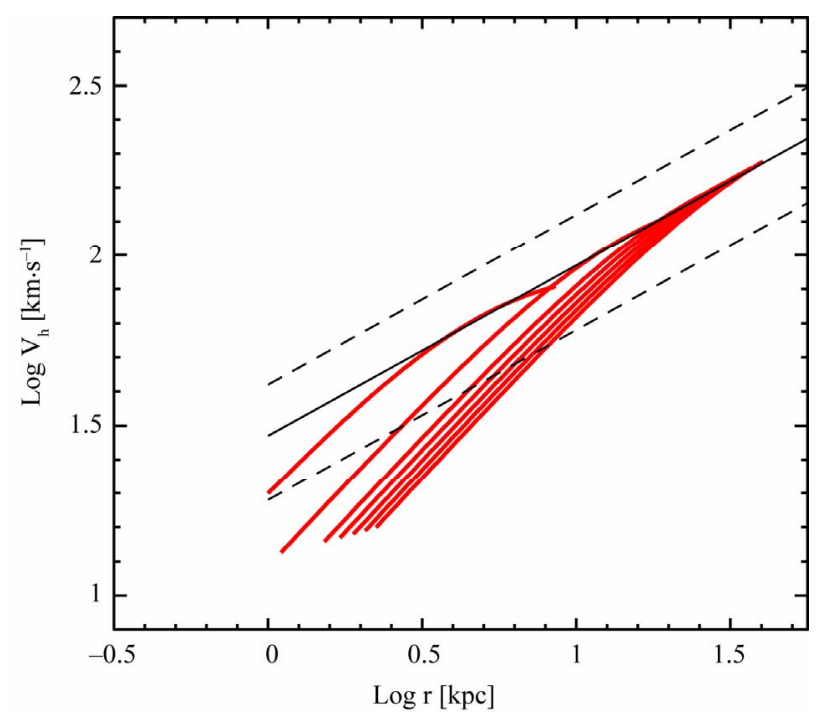

Figure 3. The $\mathrm{W}+10$ halo velocity-radius relationship (black line) with its $1-\sigma$ scatter (black dashed lines) plotted with the URCH profiles (red lines). assumptions adopted in the mass modelling. Moreover, the URCH profiles are obtained from hundreds of RCs, while $V_{h}(r)$ in $\mathrm{W}+10$ only from few $($ see $\mathrm{W}+10)$.

As a result, in a first approximation we can claim that the $\mathrm{W}+10$ relationship, in the radial range $1 \mathrm{kpc}$ $\leq r \leq 30 \mathrm{kpc}$, is a projection, on the $\left(r, V_{h}(r)\right)$ axes of a more complex $V_{U R C}\left(r / R_{\text {opt }}, L\right)$ relationship in which the structural quantities $V_{U R C}(1, L), L$ and $R_{D}$ are related in a specific way. This suggests the existence of a number of scaling laws between disk scalenghts, disk mass, halo structural parameters and galaxy luminosity, which by the way have been also observed through the analysis of accurate mass models in large samples of galaxies (e.g. $\mathrm{S}+07)$.

The $\mathrm{W}+10$ relation URC-supported, becomes very useful to test whether DM halos in spirals are consistent with NFW density profile. In this case let us remind that (see [19]):

$$
V_{N F W}^{2}(r)=V_{v i r}^{2} \frac{g(c)}{x g(c x)}
$$

where $x=r / R_{v i r}$ is the radial coordinate, $g(c)=[\ln (1+c)-c /(1+c)]^{-1}$, and for the concentration parameter $c$ we take

$$
c\left(M_{v i r}\right)=9.2\left(\frac{M_{v i r}}{10^{12} h^{-1} M_{\odot}}\right)^{-0.09}
$$

(see $[1,20])$. The halo mass inside a radius $r$ is given by:

$$
M_{N F W}(x)=M_{v i r} \frac{g(c)}{g(c x)}
$$

In Figure 4 we plot $V_{N F W}\left(r, M_{v i r}\right)$ for the radial and halo mass ranges identical with those investigated above (more specifically we consider 8 masses equally logspaced in the range: $\left.7.7 \times 10^{11} \leq M_{v i r} / M_{\odot} \leq 1.1 \times 10^{13}\right)$.

The $\mathrm{W}+10$ halo velocity-radius relationship (black line) with its $1-\sigma$ scatter (black dashed lines) plotted with 1) the halo velocities of our Sample A of 116 spirals (black points), 2) the NFW halo velocity profiles (blue lines, with the thick curves corresponding to objects with mass comparable with those of the individual Sample).

The resulting halo velocity curves $V_{N F W}(r)$ occupies a very well defined portion in the $\left(r, V_{h}(r)\right)$ plane (blue lines). However, this region is mostly off that defined by 1) the $\mathrm{W}+10$ relationship, 2) the similar relationship derived in this paper by a larger number of individual RCs (of low luminosity objects) and 3) a specific projection of the URCH (given in Figure 2). A disagreement of the NFW halo velocity profiles with the actual kinematics of spirals is not new (e.g. [21]). However, here, we make a step further by investigating an unprecedented large number of low luminosity objects. We de- 
rive their $V_{h}(r)$ profiles from disk kinematics by adopting only weak and reasonable assumptions. These profiles result incompatible with the NFW profiles for any value of their masses and their concentrations.

\section{DISCUSSION}

We have compared the $\mathrm{W}+10\left(V_{h}\left(R_{1 / 2}\right)-R_{1 / 2}\right)$ relationship (see also $M+07$ ), with the URCH and with the kinematics of 116 spirals of low luminosity objects. We find that the $\mathrm{W}+10$ "unique" velocity profile is essentially in agreement with the URC paradigm of which it is a sort of projection. The $\mathrm{W}+10$ relation alongside its not negligible scatter of $0.25 \mathrm{dex}$, can be identified as the projection of the URC on the $\left(r, V_{h}(r)\right)$ plane. This implies the existence of a number of scaling laws between disk scalenghts, disk mass, halo structural parameters and galaxy luminosity, observed also analyzing accurate mass models in large samples of galaxies.

We claim that the distribution of dark and luminous matter in galaxies is such that at any chosen radius $r$, $V_{h}(r)$ takes, in different galaxies of different mass, almost the same value. The simplest explanation is that the more massive is the dark halo, the lesser dense and concentrated it turns out to be. The net effect is that the dark mass inside a chosen physical radius is weakly dependent of the total halo mass. The physical explanation of this still lags: we realize that the dark and the luminous matter are distributed in a related way, which is not obvious, in view of their very different nature. It is worth to point out that the URCH profiles for virial masses $M_{v i r}>3 \times 10^{12} M_{\odot}$, are discrepant with the $\mathrm{W}+$ 10 relationship, also considering its (quite large) scatter (see Figure 3). Although in the $\mathrm{W}+10$ sample there is an obvious shortage of such big objects, it would seem that for the about 5\% top luminous objects, the halo velocity profile implied by $\mathrm{W}+10$ does not agree with the URC mass model and it indicates a larger amount of DM. This issue will be clarified only with a bigger sample of individual RCs. At low masses, low circular velocities and low radii (1 kpc $<\mathrm{r}<30 \mathrm{kpc})$, we find the existence of an "unique" $V_{h}(r)$ profile whose physical meaning has to be understood. This is amazing in that by adopting the radial units, i.e. $\mathrm{kpc}$, we lose any autosimilarity of the velocity/mass profiles, present when the radial coordinate is measured in terms of $R_{v i r}$ or $R_{D}$. We can use the $\mathrm{W}+10$ relationship as a Cosmology Test: in fact, when plotted in radial physical units, the NFW velocity profiles lie in a very small region, almost independently of their halo masses and concentration. Such a region, however, turns out to be clearly outside that indicated by the derived halo velocity profiles in $\mathrm{W}$ +10 , in the URCH and in our sample of individual RCs. Especially at low luminosities, where the NFW velocities are $0.2-0.4$ dex higher, the observed discrepancy is very

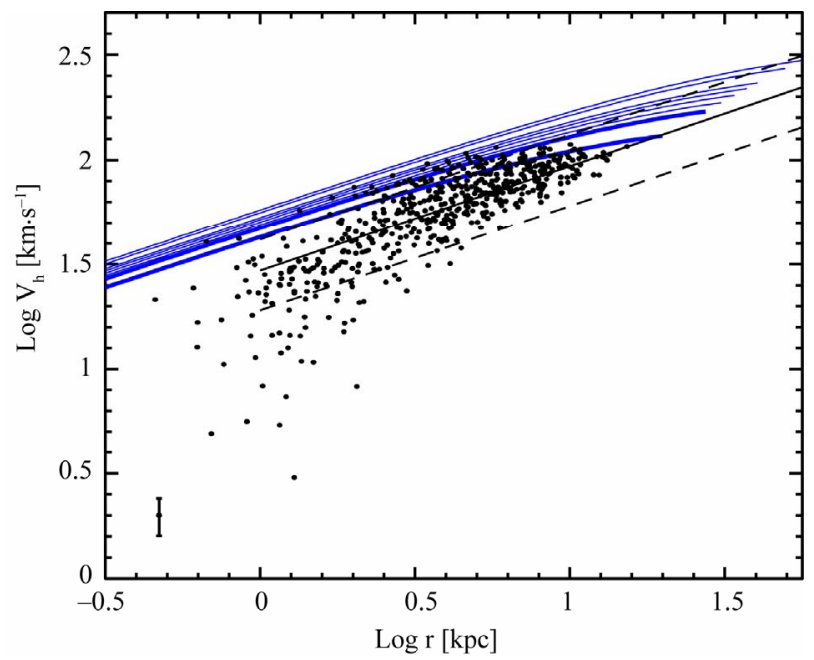

Figure 4. The $\mathrm{W}+10$ halo velocity-radius relationship (black line) with its $1-\sigma$ scatter (black dashed lines) plotted with 1) the halo velocities of our Sample A of 116 spirals (black points), 2) the NFW halo velocity profiles (blue lines, with the thick curves corresponding to objects with mass comparable with those of the individual Sample).

severe in that, being the contribution of the luminous matter quite negligible, $V_{h}(r)$ is virtually derived in a model independent way, and it almost coincides with the observed $V(r)$. Of course, the inconsistency between RCs and NFW halo + disk mass model is already well known, even if it is proved only in a quite limited number of test cases ([22]). Here we have opened the possibility to investigate such a crucial issue in direct way and by means of a large number of objects. It is worth recalling that also within $\Lambda \mathrm{CDM}$ scenario there are several ways to modify the original NFW halo profiles to be compatible with observations, see [23-25] and references therein.

\section{REFERENCES}

[1] Salucci, P., Martins, C.F. and Lapi, A. (2011) DMAW 2010 LEGACY the presentation review: Dark matter in galaxies with its explanatory note. arXiv:1102.1184v1.

[2] Walker, M.G., McGaugh, S.S., Mateo, M., Olszewski, E.W. and Kuzio de Naray, R. (2010) Comparing the dark matter halos of spiral, low surface brightness, and dwarf Spheroidal galaxies. Astrophysical Journal Letters, 717, L87-L91. doi:10.1088/2041-8205/717/2/L87

[3] McGaugh, S.S., De Blok, W.J.G., Schombert, J.M., Kuzio de Naray, R. and Kim, J.H. (2007) The rotation velocity attributable to dark matter at intermediate radii in disk galaxies. Astrophysical Journal, 659, 149-161. doi:10.1086/511807

[4] Persic, M. and Salucci, P. (1991) The universal galaxy rotation curve. Astrophysical Journal, 368, 60-65. doi:10.1086/169670

[5] Persic, M., Salucci, P. and Stel, F. (1996) The universal 
rotation curve of spiral galaxies-I. The dark matter connection. Monthly Notices of the Royal Astronomical Society, 281, 27-47.

[6] Catinella, B., Giovanelli, R. and Haynes, M.P. (2006) Template rotation curves for disk galaxies. Astrophysical Journal, 640, 751-761. doi:10.1086/500171

[7] Rhee, G., Valenzuela, O., Klypin, A., Holtzman, J. and Moorthy, B. (2004) The rotation curves of dwarf galaxies: A problem for cold dark matter? Astrophysical Journal, 617, 1059-1076. doi:10.1086/425565

[8] Salucci, P., Lapi, A., Tonini, C., Gentile, G., Yegorova, I. and Klein, U. (2007) The universal rotation curve of spiral galaxies-II. The dark matter distribution out to the virial radius. Monthly Notices of the Royal Astronomical Society, 378, 41-47. doi:10.1111/j.1365-2966.2007.11696.x

[9] Persic, M. and Salucci, P. (1995) Rotation curves of 967 spiral galaxies. Astrophysical Journal Supplement, 99, 501. doi:10.1086/192195

[10] Freeman, K.C. (1970) On the disks of spiral and so galaxies. Astrophysical Journal, 160, 811. doi:10.1086/150474

[11] Persic, M. and Salucci, P. (1990) Mass decomposition of spiral galaxies from kinematics. Monthly Notices of the Royal Astronomical Society, 245, 577.

[12] Evoli, C., Salucci, P., Lapi, A. and Danese, L. (2011) The $\mathrm{HI}$ content of local late-type galaxies. Astrophysical Journal, 743, 45. doi:10.1088/0004-637X/743/1/45

[13] Eke, V.R., Cole, S. and Frenk, C.S. (1996) Cluster evolution as a diagnostic for Omega. Monthly Notices of the Royal Astronomical Society, 282, 263-280.

[14] Donato, F., Gentile, G., Salucci, P., Frigerio Martins, C., Wilkinson, M.I., Gilmore, G., Grebel E.K., Koch, A., and Wyse, R. (2009) A constant dark matter halo surface density in galaxies. Monthly Notices of the Royal Astronomical Society, 397, 1169-1176. doi:10.1111/j.1365-2966.2009.15004.x

[15] Shankar, F., Lapi, A., Salucci, P., De Zotti, G. and Danese, L. (2006) New relationships between galaxy properties and host halo mass, and the role of feedbacks in galaxy formation. Astrophysical Journal, 643, 14-25.

\section{doi:10.1086/502794}

[16] Yegorova, I.A. and Salucci, P. (2007) The radial TullyFisher relation for spiral galaxies I. Monthly Notices of the Royal Astronomical Society, 377, 507-515. doi:10.1111/j.1365-2966.2007.11637.x

[17] Persic, M. and Salucci, P. (1990) The disk contribution to rotation curves of spiral galaxies. Monthly Notices of the Royal Astronomical Society, 247, 349.

[18] Salucci, P., Ashman, K. and Persic, M. (1991) The dark matter content of spiral galaxies. Astrophysical Journal, 379, 89-93. doi:10.1086/170485

[19] Navarro, J.F., Frenk, C.S. and White, S.D.M. (1996) The structure of cold dark matter halos. Astrophysical Journal, 462, 563. doi:10.1086/177173

[20] Klypin, A.A., Trujillo-Gomez, S. and Primack, J. (2011) Dark matter halos in the standard cosmological model: Results from the bolshoi simulation. The Astrophysical Journal, 740, 102. doi:10.1088/0004-637X/740/2/102

[21] Gentile, G., Salucci, P., Klein, U., Vergani, D. and Kalberla, P. (2004) The cored distribution of dark matter in spiral galaxies. Monthly Notices of the Royal Astronomical Society, 351, 903-922. doi:10.1111/j.1365-2966.2004.07836.x

[22] Salucci, P. and Frigerio Martins, C. (2009) The mass distribution in Spiral galaxies. EAS Publications Series, 36, 133-140. doi:10.1051/eas/0936018

[23] Maccio, A.V., Stinson, G., Brook, C.B., Wadsley, J., Couchman, H.M.P., Shen, S., Gibson, B.K. and Quinn, T. (2012) Halo expansion in cosmological hydro simulations: towards a baryonic solution of the cusp/core problem in massive spirals. The Astrophysical Journal Letters, 744, L9. doi:10.1088/2041-8205/744/1/L9

[24] Governato, F., Brook, C., Mayer, L., Brooks, A., Rhee, G., Wadsley, J., Jonsson, P., Willman, B., Stinson, G., Quinn, T. and Madau, P. (2010) Bulgeless dwarf galaxies and dark matter cores from supernova-driven outflows. $\mathrm{Na}$ ture, 7278, 203-206. doi:10.1038/nature08640

[25] Tonini, C., Lapi, A. and Salucci, P. (2006) Angular momentum transfer in dark matter halos: Erasing the cusp. The Astrophysical Journal, 649, 591-598. doi:10.1086/506431 